\title{
Optimal Design and Manufacture of Active Rod Structures with Spatially Variable Materials
}

\author{
Oliver Weeger, Yue Sheng Benjamin Kang, Sai-Kit Yeung, Martin L. Dunn \\ Singapore University of Technology and Design \\ SUTD Digital Manufacturing and Design Centre \\ 8 Somapah Road, Singapore 487372, Singapore \\ oliver_weeger@sutd.edu.sg, benjamin_kang@mymail.sutd.edu.sg, \\ saikit@sutd.edu.sg, martin_dunn@sutd.edu.sg
}

\begin{abstract}
We present a design optimization and manufacturing approach for the creation of complex 3D curved rod structures with spatially variable material distributions that exhibit active deformation behavior, enabled by the shape memory effect of 3D printed photopolymers - so-called 4D printing. Our framework optimizes the crosssectional properties of a rod structure, in particular the Young's modulus, such that under given loading conditions it can obtain one or more target shapes resulting from geometrically nonlinear deformation, from which the structure can then actively deform back to the original shape due to the shape memory effect. Our approach includes a novel algorithm to generate physical realizations from the computational design model, which allows their direct fabrication via printing of shape memory composites with voxel-level compositional control with a multi-material 3D printer. Our design and manufacture digital toolchain allows the continuous variation of multiple active materials as a route to optimize mechanical as well as active behavior of a structure, without changing the original shape of the $3 \mathrm{D}$ rod structure, which is not possible with a single material. We demonstrate the entire design-fabrication-test approach and illustrate its capabilities with examples including 3D characters, personalized medical applications, and complex structures that exhibit instabilities during their nonlinear deformation.
\end{abstract}

\section{Keywords}

4D printing, design optimization, voxel control of material properties, active structures, multi-material 3D printing, functionally graded materials.

\section{Introduction}

Digital design and manufacturing is a rapidly growing field of research and application, as new ways to digitize design and manufacturing workflows are emerging and enabling products with new and/or optimized functionality, faster prototyping of virtual designs into physical artifacts, and direct production of parts. A particularly attractive paradigm is the ability to design and manufacture components based on the control of the composition of multiple materials at the scale of micrometer-size voxels in a $3 \mathrm{D}$ volume.

The creation of digital composite materials and components is also being enabled by simultaneous advances in active materials such shape memory polymers that can be switched between multiple equilibrium configurations by an environmental stimulus, e.g. a temperature change, without application of external forces, see Fig. 1. When this controllable, nonlinear, active behavior is enabled by 3D printing of digital shape memory polymers (SMP) and composites (SMC), it is called $4 D$ printing. These new possibilities in manufacturing and functionality call for the development of computational design methods and software, that allow designers, engineers and architects to virtually explore and optimize their drafts with respect to various kinds of design variables, such as shape, material and functionality, and then immediately physically realize them through $3 \mathrm{D}$ printing.

The general idea of manufacturing active structures or smart materials by 3D printing, so-called $4 D$ printing, was first proposed based on swelling gels ${ }^{1}$ and then demonstrated using multiple photopolymers, exploiting the shape memory effect (SME) ${ }^{2}$. More recently, 4D printing was extended to more complex self-folding structures, so-called active origami ${ }^{3}$ and to sequential self-folding structures ${ }^{4}$ as well as structures with micron-scale control 
Initial/printed shape

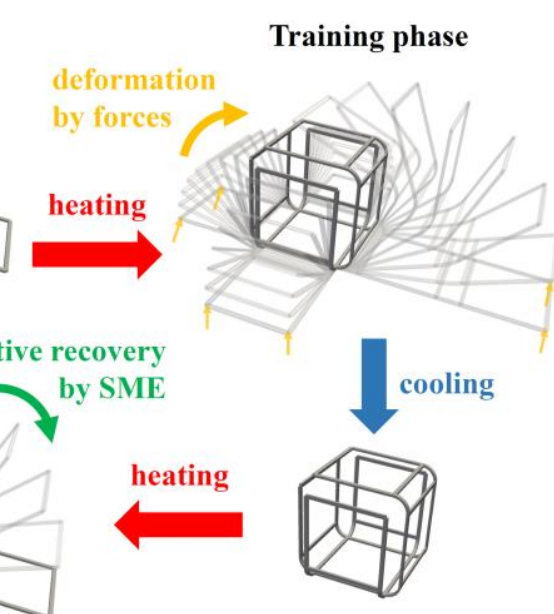

Target shape

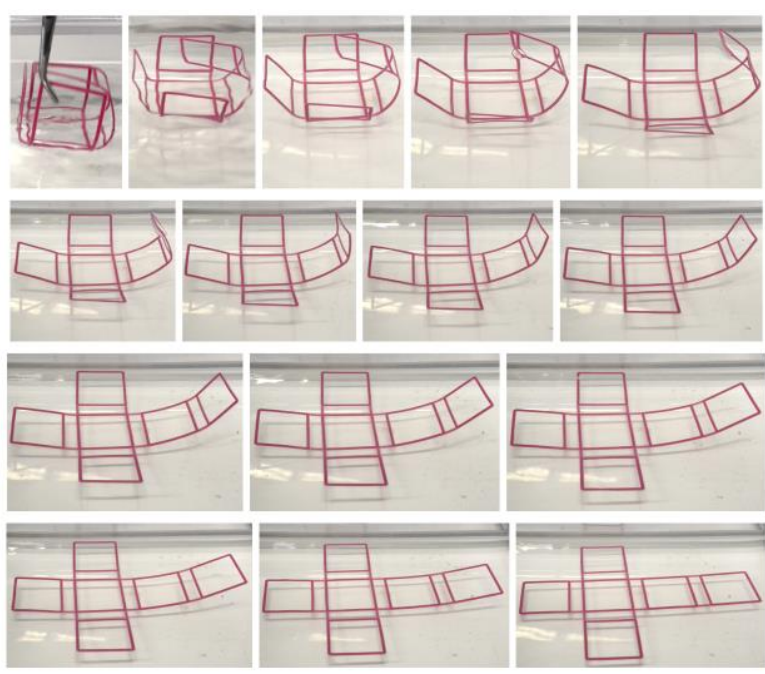

Active shape recovery

Figure 1: Illustration of shape memory process of a self-unfolding box. (a) The initially flat structure is heated to $60^{\circ} \mathrm{C}$ inside a water bath to make it more compliant; then it can be easily deformed into the desired box shape. At the end of the training phase, the box structure is cooled to room temperature and maintains its target shape due to the shape memory effect. Re-heating results in an active shape change behavior from the target box configuration back to the flat initial shape. (b) Snapshots of the active shape recovery are showing the deformation from the trained target pose (box shape, top left) to the printed initial shape (flat, bottom right).

of single and multiple materials enabled by projection microstereolithography ${ }^{5,6}$. Active behavior can also be triggered by thermal expansion mismatch ${ }^{7}$ and recently, a novel biomimetic $4 \mathrm{D}$ printing technique based on the swelling of hydrogels was presented ${ }^{8}$, as well as an approach that uses both SMPs and hydrogels to eliminate the need for mechanical training. Computational optimization approaches for $4 \mathrm{D}$ printing of freeform surfaces ${ }^{9}$ and to topology optimization for printed active composites ${ }^{10}$ have been studied, but not using spatially variable, gradient material designs.

Previous design and manufacturing methods for heterogeneous multi-material 3D printing include data-driven approaches ${ }^{11}$, as well as direct meshed-based methods ${ }^{12}$. Furthermore, multi-material design and additive manufacturing has also been combined with conventional manufacturing techniques ${ }^{13}$. In a more general setup, a fabrication algorithm that translates user-defined model specifications into material assignments was created ${ }^{14}$ and a pipeline for multi-material 3D was also presented ${ }^{15}$. Mechanical simulation ${ }^{16}$ and topology optimization methods ${ }^{17,18}$ for structures and mechanism have also been studied. Multi-material design optimization of lattice structures is also related to our approach ${ }^{19}$, as well as optimization of cross-sectional profiles of single material rod meshes ${ }^{20}$.

In this paper, we present a digital process flow from design optimization to graded multi-material manufacturing of active structures that use shape memory polymer composites to switch between multiple equilibrium configurations. We focus on complex structures made from 3D curved rods since these structures can be made to be highly deformable and are thus well-suited for large active nonlinear deformation behavior. Rod structures are versatile, and thus our approach can also contribute to the creation of active lattice structures or scaffolds for surfaces and solids.

The main objective of our approach is to allow a designer to define initial (as-printed) and target (trained) poses of a 3D rod structure, which specify two configurations that the structure can be switched between when heated. Given these shapes and corresponding loading conditions, we formulate and solve a nonlinear optimization problem that determines the spatially varying material distribution of the Young's modulus, such that the error between the actual deformation and the target configuration is minimized. We then develop algorithms to realize these objects by $3 \mathrm{D}$ printing with multi-material composition variations at the voxel scale. Though we $3 \mathrm{D}$ print the physical realizations using shape memory polymer composites, our design framework is more general and could be easily applied to other active materials as well.

\section{Design optimization and manufacturing framework}

The overall design-manufacturing process to create $3 \mathrm{D}$ rod structures with desired active shape change behavior can be summarized in a number of steps, which we briefly outline here and then describe in more detail in the following sections. This overall pipeline is illustrated in Fig. 2.

a) Modeling rod structures and simulating their mechanical deformation behavior are essential capabilities that form the foundation of our design optimization approach. The mechanics of 3-dimensional rods and rod 


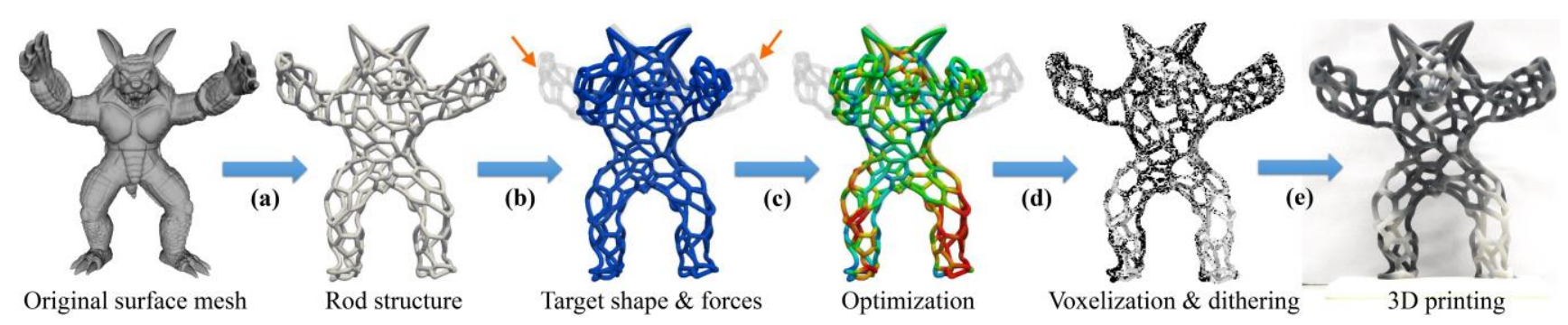

Figure 2: Outline of overall pipeline based on the Armadillo use case. A surface mesh is converted into a rod structure, then forces and a target shape are defined for the shape memory training phase, and subsequently the material distribution in terms of Young's modulus is optimized to match deformation with the target shape. Finally, the rod mesh with optimized material distribution is converted into a voxellevel representation and $3 \mathrm{D}$ printed.

structures are described by the Cosserat rod model 21. For the numerical discretization and simulation of the rods we use an isogeometric method ${ }^{22}$, which allows a direct integration of our framework into CAD environments.

b) Then a loading conditions and a target shape have to be defined. Here, activeness is realized via 4D printing shape memory polymers and composite material designs ${ }^{3}$, that allow our rod structures to actively deform back from this target shape to the original printed shape when subject to an external stimulus such as heating.

c) Our goal is to optimize for a spatially varying material composition of the rods such that it is possible to deform the rod structure into the target shape during the training phase by a simple set of forces. We formulate a nonlinear optimization problem for the material distribution in terms of Young's moduli, where the objective is to minimize the deviation of the deformed shape from the target shape, which is constrained by the mechanics of the rod structure. Optimization of the material distribution enables the designer to create an initial design, which won't change its visual appearance during the optimization process, as opposed to classical shape and topology optimization approaches.

d) The final step is the realization of the optimized designs by a multi-material 3D printer. This requires a multi-material printing pipeline for material distributions with spatially variable stiffness: we first voxelize the optimized rod structure and then translate the continuous Young's modulus distributions into material ratios by a modulus-to-material mapping. Since each voxel can only contain one drop of a unique model material, dithering has to be applied to generate the model material distributions, which are then output as bitmaps files for each layer of material and transferred to the printer.

e) After printing and removing the support material, our rod structures can be trained by heating and applying the design loads, cooling back to room temperature while maintaining the deformed configuration, and then removing the loads. Then we test them to verify their active shape change behavior.

\section{Methods}

\section{Modeling of the shape memory behavior}

Exploitation of the shape memory effect requires the following process a) fabricating a component from a suitable SMP so that it assumes its primary shape at room temperature, b) programming it by heating it above its transition temperature, applying prescribed mechanical loads to deform it into a desired secondary shape, and then cooling it to room temperature while maintaining the loads or the shape, at which point it temporarily resides in its secondary shape, and then c) deploying the component to actively recover its primary shape by heating it above its transition temperature (see Fig. 1). The performance of an SMP material and/or component is described by two parameters: a) fixity is the degree to which the deformed shape is retained after cooling and release of loads, and b) recovery is the degree to which the actual primary shape is recovered during deployment.

Here we develop a design and manufacturing workflow for components constructed by rod meshes that can transform between a printed primary shape and a target secondary shape. The shape memory behavior of the rod structure is enabled by fabricating them with shape memory composites (SMCs) that exhibit tunable shape memory behavior that can vary along the axis of each rod. These spatially dependent layouts are realized by the voxel-level control of mixtures of two polymers as determined by optimal design of SMCs with microstructures at a scale an order of magnitude smaller than the rods themselves based on a rigid polymer and an elastomer. In order to simplify the design problem, we ignore the time-dependence of the fixity and recovery processes of the composites in the rod structure and assume that they are perfect, i.e. 100\%. This reduces the design problem to determining the spatial distribution of the nonlinear elasticity (realized by an underlying composite microstructure) within a rod mesh at the elevated training temperature. This is significant because even through our simplified computational design problem we still exploit the sophisticated shape memory properties of fixity and recovery. 


\section{Modeling, simulation and design of rod structures}

A rod is a slender, i.e. long and thin, 3-dimensional deformable body - its length being significantly longer than its cross-section diameter. For the mechanical modeling of relatively thick elastic rods we use the nonlinear Cosserat rod theory ${ }^{21}$. It is based on the description of the configuration of the rod as a framed curve, i.e. a rod is represented by the line of its mass centroids, its centerline, which is a spatial curve $\boldsymbol{r}(\mathrm{s}):[0, \mathrm{~L}] \rightarrow \mathbb{R}^{3}$ and a frame or triad $\boldsymbol{R}(\mathrm{s}):[0, \mathrm{~L}] \rightarrow \mathrm{SO}(3)$, which describes the evolution of the orientation of the cross-sections along the centerline and can be associated with a rotation matrix $\boldsymbol{R}(s)=\left(\boldsymbol{d}_{1}(s), \boldsymbol{d}_{2}(s), \boldsymbol{d}_{3}(s)\right) \in \mathbb{R}^{3 \times 3}: \boldsymbol{R}^{\top} \boldsymbol{R}=\boldsymbol{I}$.

This representation of a rod using its centerline curve and frames completely determines its kinematic configuration, which is governed by the equilibrium equations of linear and angular momentum, $\boldsymbol{n}^{\prime}+\widehat{\boldsymbol{n}}=\mathbf{0}$ and $\boldsymbol{m}^{\prime}+\boldsymbol{r}^{\prime} \times \boldsymbol{n}+\widehat{\boldsymbol{m}}=\mathbf{0}$, as well as boundary conditions. Here, $\boldsymbol{n}=\boldsymbol{R} \boldsymbol{\sigma}$ and $\boldsymbol{m}=\boldsymbol{R} \boldsymbol{\chi}$ represent the internal forces and moments of the rod. External forces and moments are given by $\widehat{\boldsymbol{n}}$ and $\widehat{\boldsymbol{m}}$. The stresses $\boldsymbol{\sigma}=\boldsymbol{C} \boldsymbol{\varepsilon}$ and $\chi=\boldsymbol{D} \boldsymbol{\kappa}$ are determined through linear constitutive laws using the strains $\boldsymbol{\varepsilon}=\boldsymbol{R}^{\top} \boldsymbol{r}^{\prime}-\boldsymbol{e}_{3}$ and $\boldsymbol{\kappa}=\left[\boldsymbol{R}^{\prime \top} \boldsymbol{R}\right]_{\times}$. The geometric and material properties of the rod cross-sections enter the formulation through the constitutive matrices $\boldsymbol{C}$ and $\boldsymbol{D}$, which both depend on the Young's modulus $E$.

For the computational solution of the governing equations of the Cosserat rod model we use an isogeometric ${ }^{23}$ collocation method ${ }^{22}$. It provides an accurate and efficient numerical discretization of the model and enables a seamless integration of the design-to-manufacturing pipeline through a consistent representation of geometry using NURBS curves: $\boldsymbol{r}(s)=\sum_{i=1}^{n} N_{i}(s) \boldsymbol{r}_{i}$. Here $N_{i}$ are the $n$ NURBS basis functions and $\boldsymbol{r}_{i}$ the control points of the curve. The rotation matrices are parameterized as $\boldsymbol{R}(s) \equiv \boldsymbol{R}(\boldsymbol{q}(s)$ ) with unit quaternions $\boldsymbol{q}(s)=$ $\sum_{i=1}^{n} N_{i}(s) \boldsymbol{q}_{i},\|\boldsymbol{q}(s)\|=1$.

Within the isogeometric collocation framework, these NURBS discretizations of centerline and rotation quaternions are substituted into the governing equations of the rod model and evaluated at $n$ so-called collocation points ${ }^{24}$. This determines a nonlinear system of equations $\boldsymbol{f}(\overrightarrow{\boldsymbol{r}}, \overrightarrow{\boldsymbol{q}})=\mathbf{0}$ for the unknown vectors $\overrightarrow{\boldsymbol{r}}=\left(\boldsymbol{r}_{i}\right)_{i=1, \ldots, n}$ and $\overrightarrow{\boldsymbol{q}}=\left(\boldsymbol{q}_{i}\right)_{i=1, \ldots, n}$, which has to be solved with a Newton's method in order compute the deformed configuration of a rod. For the extension from a single rod to rod structures, i.e. meshes of interconnected rods, a rigid coupling of the rods is enforced 22 .

The initial geometry of a rod structure serves as input for our overall framework. We support three different approaches:

a) NURBS curve mesh import from CAD. The NURBS parameterization of the rods allows an isogeometric integration of the design and analysis process, without any format conversions or loss of geometric accuracy. Thus designers or design engineers can manually draw or use procedural modeling approaches to generate NURBS curve meshes in a CAD software, which we can then directly import into our design optimization framework.

b) Boundary extraction of NURBS surfaces. Very often in industrial computer-aided design and manufacturing, objects are designed by a boundary representation using a collection of many NURBS surfaces patches. A convenient way for rod mesh generation is thus the extraction of the boundary edges of these surfaces patches, which directly provides a collection of NURBS curves. With some more pre-processing, such as removal of duplicated curves, detection of intersections and insertion of additional isocurves, these curve meshes can also directly serve as rod meshes.

c) From triangular surface meshes. Since triangle meshes are a frequent way of storing and exchanging 3D models in CAD and digital manufacturing, we have implemented a mesh generation pipeline for triangle meshes. First, the original high resolution mesh is simplified until 100-500 triangles are left using the quadric error metric method ${ }^{25}$, to which we have added a check for maintaining a manifold mesh during simplification to prevent mesh discontinuities at low triangle counts. Then the dual of the simplified mesh is constructed, mainly consisting of hexagonal shapes, which gives more freedom for deformation ${ }^{20}$. These dual lines are then subdivided, projected back onto the original mesh and interpolated by NURBS curves.

\section{Formulation and solution of the $4 D$ printing design optimization problem}

The ultimate goal of this work is to optimize the cross-sectional properties of the rods - in particular the material distribution within a rod structure - such that a desired target configuration $\left(\boldsymbol{r}_{\boldsymbol{t}}, \boldsymbol{R}_{\boldsymbol{t}}\right)$ can be achieved as 


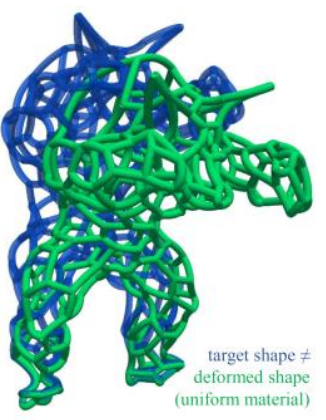

Before optimization
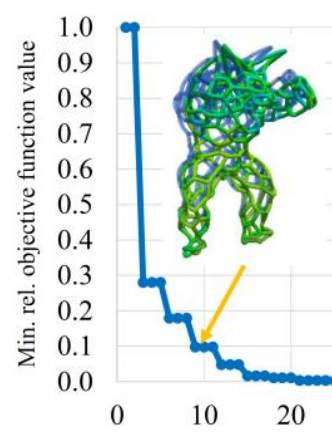

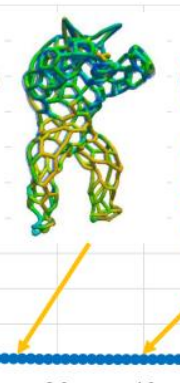

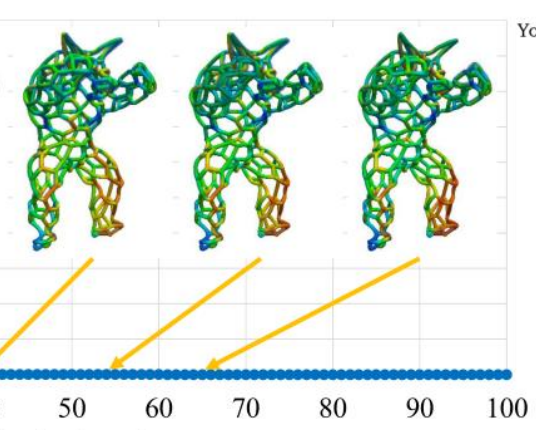

100

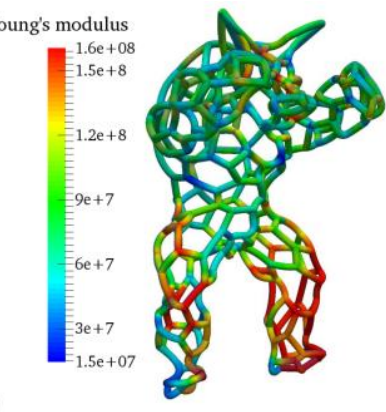

After optimization

Figure 3: Optimization process for the Armadillo use case. The deformed shape (colored by Young's modulus) comes more and more close to the target training shape (blue opaque). After 100 iterations the relative objective function value $\mathrm{g} / \mathrm{g}_{0}$ is minimized from 1.0 to $6 \cdot 10^{-5}$ and the optimized deformation and target shape have become indistinguishable.

deformation of an initial configuration $\left(\boldsymbol{r}_{\mathbf{0}}, \boldsymbol{R}_{\mathbf{0}}\right)$ during the shape memory training phase. Therefore, we first introduce a parameterization of the design variables - the material parameters of the cross-section - and then derive a suitable optimization formulation.

The essential information about the material is featured in the constitutive matrices $\boldsymbol{C}$ and $\boldsymbol{D}$ of the Cosserat rod model, which depend on the Young's modulus $E$. Typically, $E$ is overall constant for a rod, or even a whole structure, but here we take $E$ to be a design variable that can continuously vary with position and optimize its spatial dependence. Therefore, we parameterize the design variable along the centerline of the rods, i.e. $E \equiv E(s)$ for $s \in[0, L]$. Like $\boldsymbol{r}(s)$ and $\boldsymbol{q}(s)$, we discretize it according to the isogeometric concept as a NURBS curve: $E(s)=\sum_{i=1}^{n^{e}} N_{i}^{e}(s) E_{i}$. Here we are using $n^{e}$ basis functions $N_{i}^{e}(s)$, which may not necessarily be the same as $N_{i}(s)$ 's above.

We formulate a constrained nonlinear optimization problem for optimizing the Young's modulus distribution $E(s)$ such that the deformed configuration $(\boldsymbol{r}, \boldsymbol{R})$ under given boundary conditions and design loads matches the shape of the desired target configuration $\left(\boldsymbol{r}_{\boldsymbol{t}}, \boldsymbol{R}_{\boldsymbol{t}}\right)$, which is specified by the designer, as close as possible:

$$
\min _{E_{0} \leq \overrightarrow{\boldsymbol{E}} \leq E_{1}} g(\overrightarrow{\boldsymbol{E}}) \text { s.t. } \boldsymbol{f}(\overrightarrow{\boldsymbol{r}}, \overrightarrow{\boldsymbol{q}})=\mathbf{0} .
$$

Here $\overrightarrow{\boldsymbol{E}}=\left(E_{i}\right)_{i=1, \ldots, n^{e}}$ is the vector of design variables and $E_{0}$ and $E_{1}$ are the minimal and maximal Young's modulus values. The objective function $g(\overrightarrow{\boldsymbol{E}})$ measures the deviation of the deformed rod configuration from the target configuration in terms of the errors of positions $\boldsymbol{r}(\mathrm{s})$ and curvatures $\boldsymbol{\kappa}(s)$ :

$$
g(\overrightarrow{\boldsymbol{E}})=\frac{C_{r}}{2 L^{2}} \sum_{i=1}^{n}\left\|\boldsymbol{r}\left(\tau_{i}\right)-\boldsymbol{r}_{\boldsymbol{t}}\left(\tau_{i}\right)\right\|^{2}+\frac{C_{k} L^{2}}{2} \sum_{i=1}^{n}\left\|\boldsymbol{\kappa}\left(\tau_{i}\right)-\boldsymbol{\kappa}_{\boldsymbol{t}}\left(\tau_{i}\right)\right\|^{2}
$$

For the overall rod structure, target function contributions from the individual rods defined are simply added to define the global objective function. This optimization problem is then solved using an iterative nonlinear optimization solver, which evaluates the constraint $\boldsymbol{f}(\overrightarrow{\boldsymbol{r}}, \overrightarrow{\boldsymbol{q}})$ and the design sensitivities $d g / d \overrightarrow{\boldsymbol{E}}$ in each iteration. This process is illustrated in Fig. 3 for the case of an actuated Armadillo character.

\section{Multi-material 3D printing pipeline for spatially variable materials}

Once the optimal material distribution is obtained, we realize it by $3 \mathrm{D}$ multi-material printing and to do this we have created algorithms to execute the following steps as shown in Fig. 4: a) generate a voxel representation of the rod mesh; b) convert the Young's modulus value at each voxel into a volume fraction of the model materials; c) dither it into a discrete model material distribution; and d) print using a multi-material printer capable of voxellevel control of material composition (the Stratasys Objet500 Connex3 here).

Typically, solid bodies are represented as surfaces meshes, such as triangular meshes, and fast GPU-supported algorithms have been established for their voxelization ${ }^{26}$. However, the volumetric representation of a rod mesh is given in terms of centerlines with cross-section frames and shapes. Furthermore, the volume fraction occupied by the rod mesh is in general much smaller than the full printing space, since rods are slender structures. Due to the resolution of the Stratasys Objet500 3D printer, where one voxel is $42.3 \times 84.6 \times 30 \mu \mathrm{m}$, the total number of voxels is large, e.g. a $8.46 \times 8.46 \times 9.0 \mathrm{~cm}$ grid would contain 6 giga voxels, but the rod mesh would only occupy 


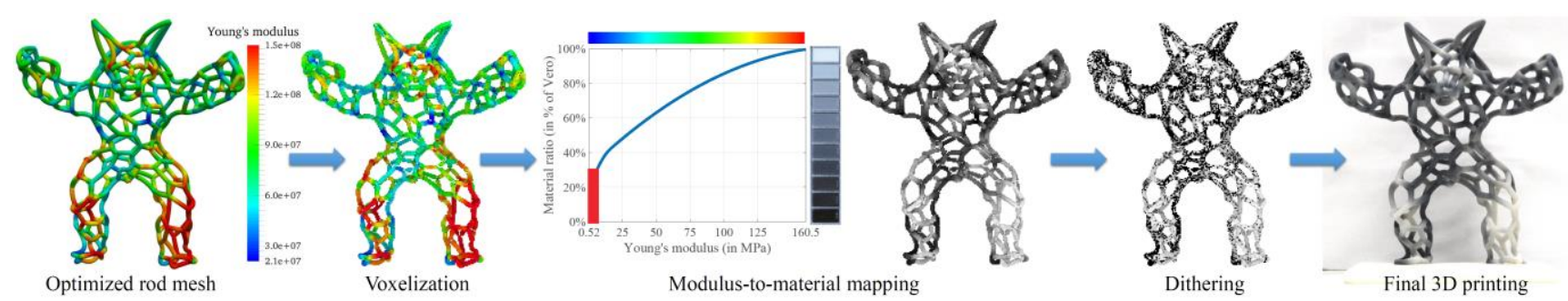

Figure 4: Outline of the multi-material 3D printing pipeline for Armadillo use case. First, the rod mesh with optimized Young's modulus distribution is converted into a voxel grid (here shown at larger voxel scale for better visibility). Then the Young's modulus values of each voxel are converted to continuous material ratios using the modulus-to-material mapping. In the dithering step a unique material is assigned to each voxel and the bitmaps for final 3D printing are generated.

a fraction of it. Thus we propose a voxelization method tailored to rod meshes: For each individual rod we iterate over its centerline $\boldsymbol{r}(s)$ in a suitable variable step size for $s$, which has to be smaller than one voxel. By evaluating the corresponding frame $\boldsymbol{R}(s)$ we obtain the representation of the cross-section. Then we iterate through the current cross-section shape and assign the modulus $E(s)$ to all voxels which are intersected by the cross-section plane. In this way we obtain a sparse representation of the voxel grid of the rod structure.

The next crucial step for printing the rod structures is to establish the relationship between the Young's modulus and actual material distributions, a modulus-to-material mapping. To this end we created samples with random distributions of two model materials (here soft/rubbery Tango, and hard/stiff Vero) with volume fractions of the hard/stiff material ranging from $0 \%$ to $100 \%$ and measured their modulus in a DMA at $60^{\circ} \mathrm{C}$, which is the temperature at which we deform structures during training. From this data we generated an approximation function to provide the required continuous material-modulus relationship for use in our optimization (see plot in the middle of Fig. 4).

Based on this material fraction, we assign a unique model material to each voxel ${ }^{27,28}$. In this dithering phase, we iterate over all layers of the voxel grid and for each layer two binary sparse matrices (bitmaps) are initialized. For every voxel with non-zero stiffness we use the above-mentioned relationship to determine the corresponding material volume ratio $\eta(E) \in[0,1]$. Then a random number $\zeta \in[0,1]$ is generated and based on it the bitmap values are assigned: If $\zeta<\eta$ the bitmap value for material 1 is set to 1 , otherwise the bitmap value for material 2 . The result of this random/white-noise dithering, or mezzo tinting procedure ${ }^{29}$, are two stacks of bitmap files for each layer, one for each base material. We use this random dithering approach here, since a high-frequency distribution without clustering gives the most adequate mixture of the two base materials.

We print our rod structures on the Stratasys Objet500 Connex3 printer, a poly jet 3D printer, that can print up to 3 different photopolymer materials simultaneously. Using its voxel-printing capability, we input models as stacks of bitmaps for each material and layer. In our setting we use the materials from the Tango and Vero families (TangoBlackPlus and VeroWhitePlus). Tango materials are rubbery elastomers, i.e. have a low Young's modulus $\left(\sim 2 \mathrm{MPa}\right.$ at $25^{\circ} \mathrm{C}, \sim 0.5 \mathrm{MPa}$ at $\left.60^{\circ} \mathrm{C}\right)$, while Vero materials are rigid polymers with a higher Young's modulus $\left(\sim 2,000 \mathrm{MPa}\right.$ at $25^{\circ} \mathrm{C}, \sim 190 \mathrm{MPa}$ at $\left.60^{\circ} \mathrm{C}\right)$. Both materials generally exhibit the shape memory effect and have temperature-dependent material properties. However, the glass transition temperature of Tango is about $13^{\circ} \mathrm{C}$, and we use the materials over the range from room temperature to $60^{\circ} \mathrm{C}$ so it behaves like an elastomer for our purposes and does not exhibit shape memory behavior. To ensure sufficient fixity at $25^{\circ} \mathrm{C}$ in our structures, we restrict the minimal Young's modulus in the optimization to $8 \mathrm{MPa}$, corresponding to a volume ratio of $30 \%$ Vero, as indicated by the red box in the modulus-to-material mapping in Fig. 4.

\section{Results and discussion}

We present the results and discuss a number of applications of our multi-material optimization and 3D printing framework for active, shape changing rod structures. For all cases we print optimized structures, train them by heating in a water bath at $60^{\circ} \mathrm{C}$, applying the appropriate mechanical loads, and cooling to room temperature while maintaining the fixed displacements and removing the loads, and then reheating the structures in the water bath at $60^{\circ} \mathrm{C}$ to demonstrate the shape change. An overview of the use cases with technical details is given in Table 1.

\section{Unfolding box}

3D printing a flat structure is faster and more economical than printing a complex, truly 3-dimensional structure. Furthermore, it also makes storage and shipping of an object easier when it is flat and can self-assemble 


\begin{tabular}{|l|r|r|r|r|}
\hline Application & \#rods & \#dofs & \#d.var. & $g / g_{0}$ \\
\hline \hline $\begin{array}{l}\text { Unfolding } \\
\text { box }\end{array}$ & 34 & $2.8 \mathrm{k}$ & 136 & 0.017 \\
\hline Armadillo & 391 & $27 \mathrm{k}$ & 1564 & 0.003 \\
\hline T-Rex & 287 & $20 \mathrm{k}$ & 1148 & 0.021 \\
\hline Arm cast & 183 & $12 \mathrm{k}$ & 488 & 0.049 \\
\hline $\begin{array}{l}\text { Buckling } \\
\text { spring }\end{array}$ & 304 & $21 \mathrm{k}$ & 1216 & 0.019 \\
\hline
\end{tabular}

Table 1: Overview of applications. Number of rods (\#rods), number of degrees of freedom of simulation model (\#dofs), number of design variables of optimization (\#d.var.), minimal relative value of objective function $\left(g / g_{0}\right)$.

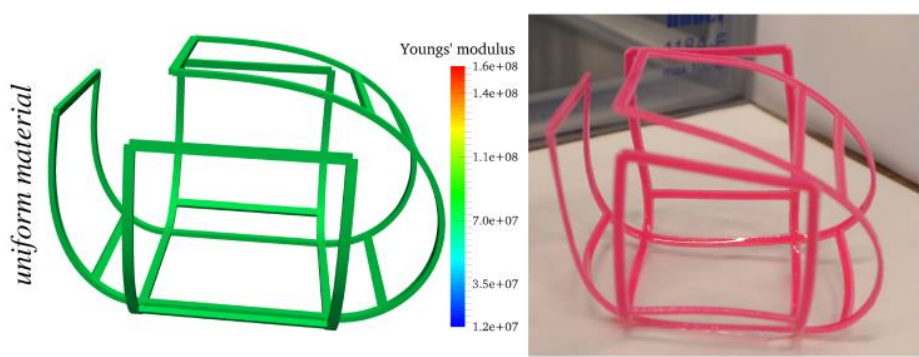

simulated training deformation

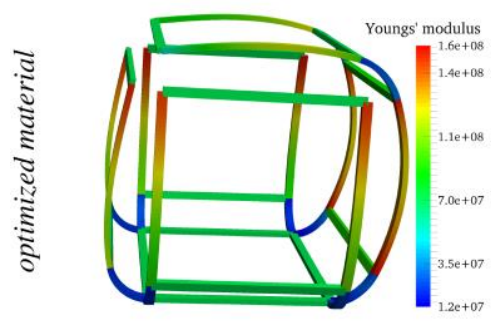

actual 3D printed structure

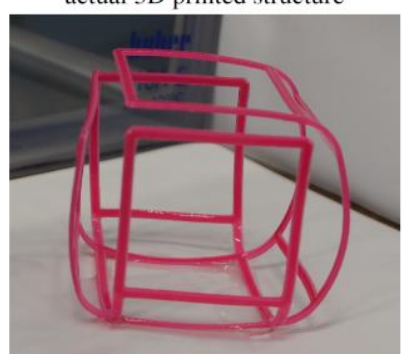

Figure 5: Unfolding box. Comparison of initial uniform and optimized material distributions and training behavior of box for computational and actual 3D printed models. The optimized structure folds much better into the desired box shape.

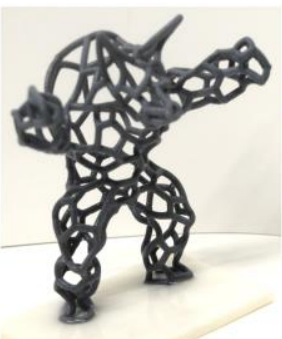

$3 \mathrm{D}$ printed structure

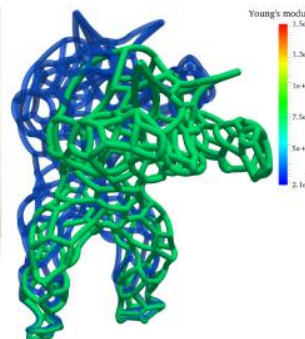

simulated training
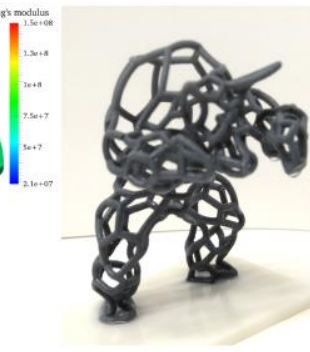

actual training with initial (uniform) material distribution

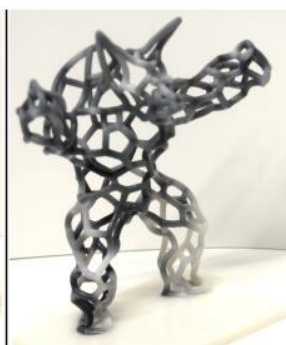

$3 \mathrm{D}$ printed structure

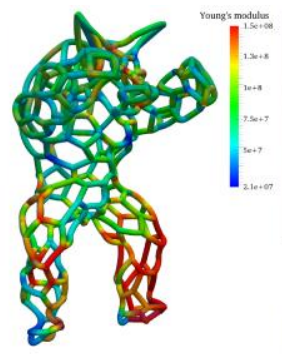

simulated training with optimized material distribution

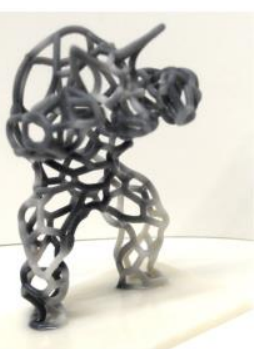

actual training
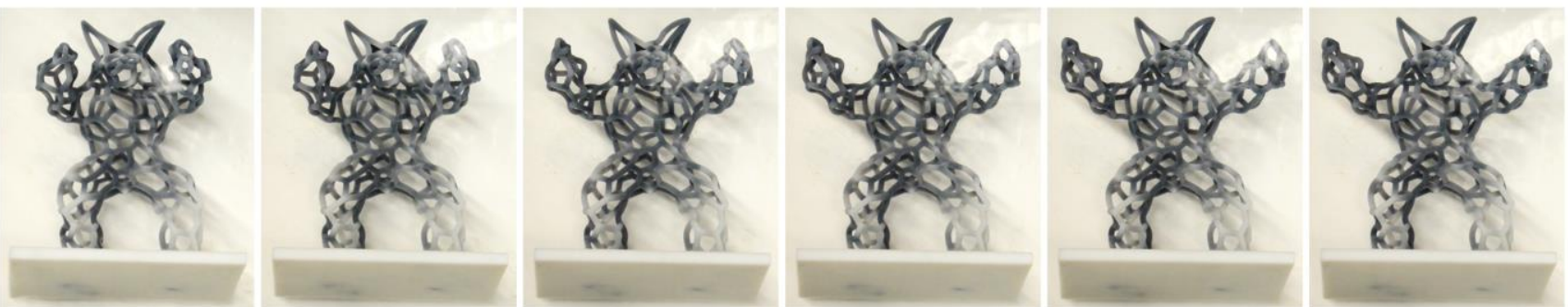

Figure 6: Armadillo. (a) Comparison of training behavior of computational and actual 3D printed models with initial uniform and optimized material distributions. While the uniform material version bends to the front during training, the optimized one remains straight. (b) Snapshots of the full active shape recovery process from deformed target (left) back to printed shape (right) inside a hot water bath.

into another desired shape. Thus we have designed a flat structure, which should be folded into a box during the training phase and unfold back as shape recovery, see Fig. 1. For the training of this very large deformation and rotation behavior, we only want to apply forces perpendicular to the outer edges of the structure, which does not result in the desired box shape with straight edges for the uniform material case. As can be seen in Fig. 5, the optimized material distribution gives a much better result, which is again validated very well by the manual training of printed structures. Snapshots of the active shape recovery from the trained box shape back to the flat initial shape are also shown in Fig. 1 (b).

\section{Armadillo}

In several explanatory figures we have already used the Stanford armadillo example. The original input mesh consists of 345,944 triangles and we have used the dual of triangle mesh procedure to generate a complex mesh with 391 curved rods. The goal is to apply a large deformation to this 3D character during the training phase such that its arms move to the front by inwards directed forces at a point on each hand, while the lower body and legs remain still. As it can be seen in Fig. 3, this is not the case for an un-optimized rod structure with uniform material distribution, but the multi-material optimization enables this desired target deformation. The comparison of training deformations of the actual 3D printed Armadillos in Fig. 6 (a) validates the computational result very well and the active shape recovery is also demonstrated in Fig. 6 (b). 

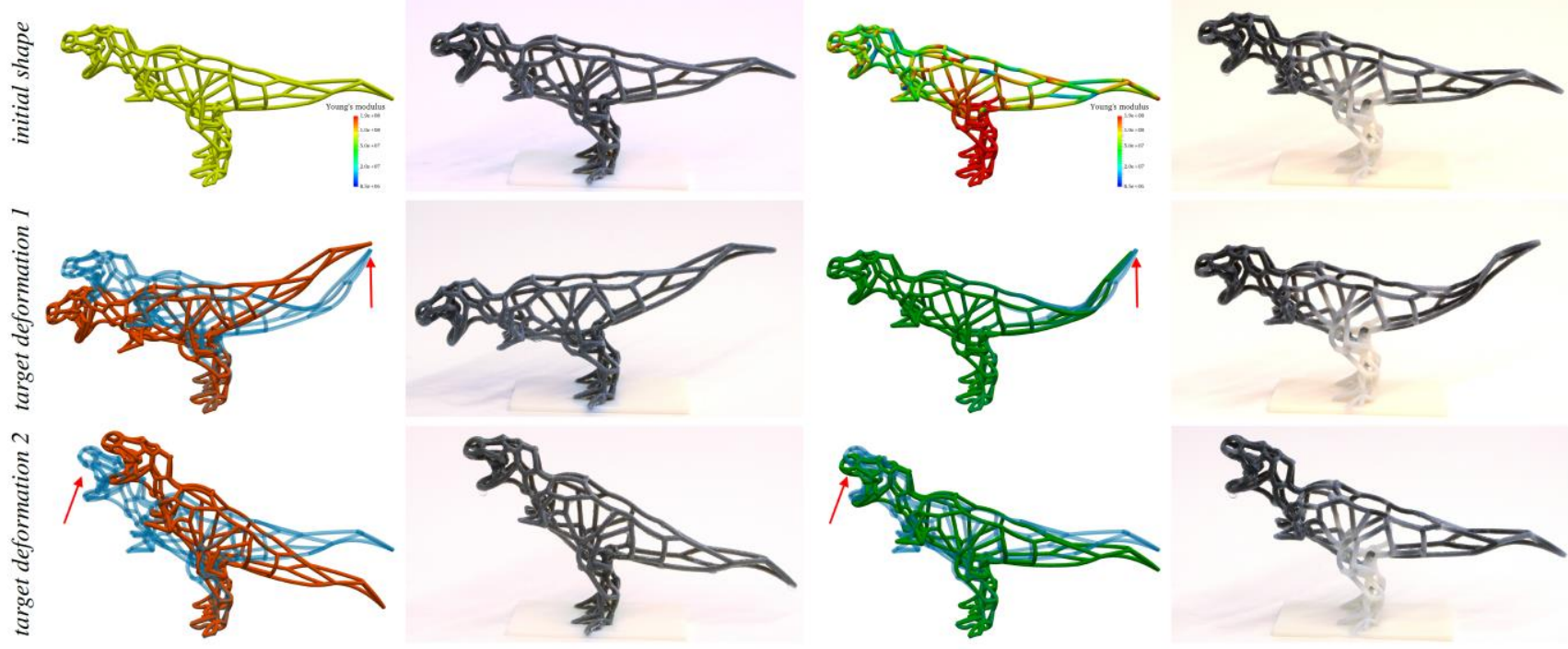

with initial (uniform) material distribution

with optimized material distribution
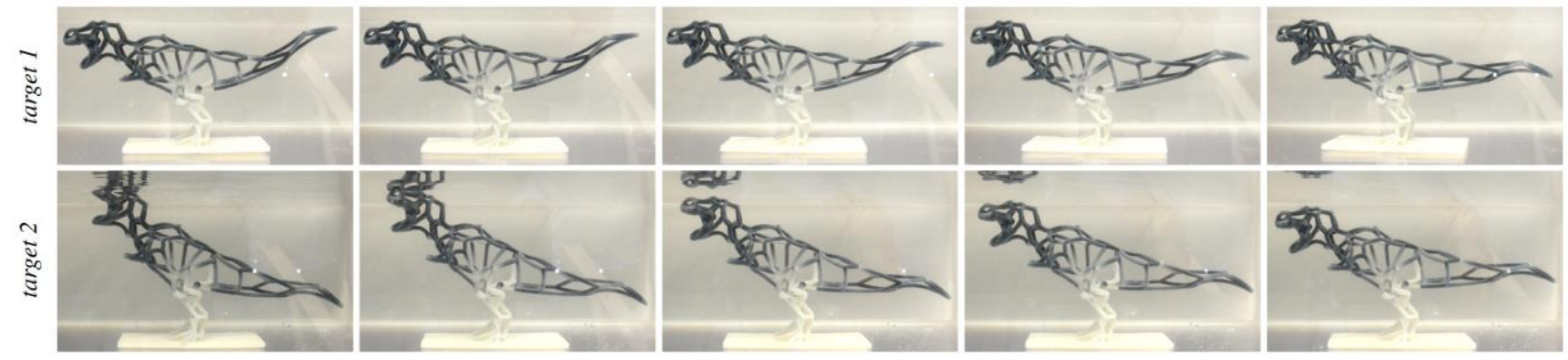

Figure 7: T-Rex. (a) Comparison of initial uniform and optimized material distributions and training behavior of dinosaur for both target shapes. The computational and actual deformations of the 3D printed models correspond very well. (b) Snapshots of active shape deformation of optimized T-Rex from both trained target poses (left) to printed initial shape (right) inside a hot water bath.

\section{$T-\operatorname{Rex}$}

Another toy-like example with a complex geometry is the Tyrannosaurus rex, see Fig. 7. The mesh with 287 curved rods is generated from an original input mesh with 9,874 triangles. For the active shape recovery behavior, we would like to train the T-Rex model with two target poses: the head moving up while the tail remains straight and vice versa. While this is not the case for the uniform material dinosaur, which just bends up and down straight, the optimized version enables both the desired target deformations - though not perfectly. Fig. 7 (b) also shows snapshots of the shape recovery process of the optimized T-Rex inside a hot water bath.

\section{Arm cast}

Next we present a practical application of $4 \mathrm{D}$ printing a medical cast for the lower arm, see Fig. 8. The cast was designed in a CAD program based on the shape of a real arm - in practice it could be a patient-specific design - and printed in its permanent cast shape. The material distribution was then optimized such that it can be flattened by hand, which allows easier storage of the cast. More importantly, a paramedic can now simply heat up the cast so it recovers back to its original shape and automatically fits around the arm of a patient, see also Fig. 8 .

\section{Buckling mechanical spring}

Finally, we present an application of a complex, repetitive mesh structure, which exhibits a very nonlinear mechanical behavior, so-called buckling. This deformation into an unstable equilibrium state, which is typically avoided in structural design and difficult to predict, is exploited here for the design of a nonlinear spring, see Fig. 9. As training shape, we define a uniform compression of the structure, resulting in buckling of the curved rods at the vertical center. When the training is done by applying a prescribed displacement on the top of the structure at four equidistant locations, this results in a different buckling pattern for the uniform material case, but our optimized structure exhibits the desired buckling in the middle. For this example, we have also verified our results by executing a compression test using mechanical testing equipment, see Fig. 9 (b). 

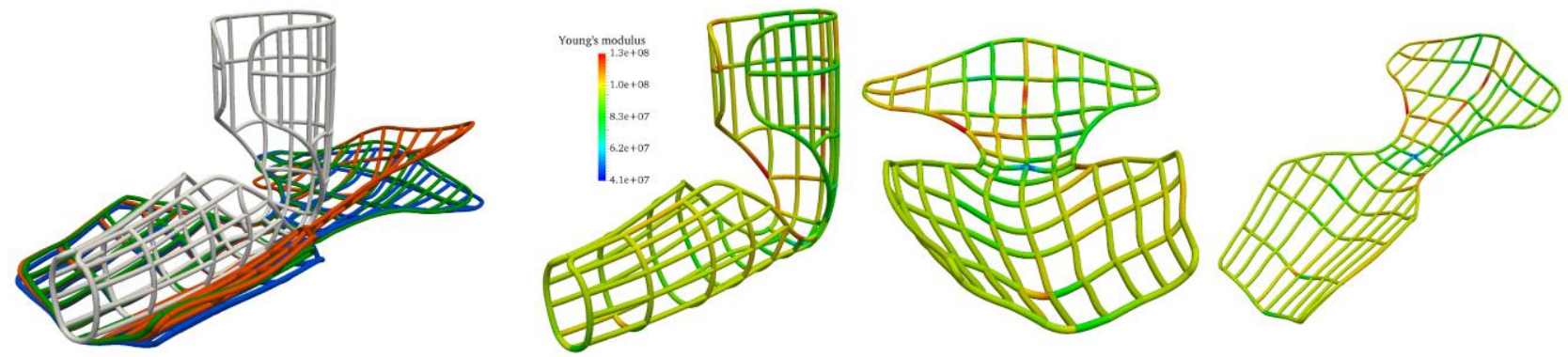

optimization result (initial, target, unopt., opt.) optimized printed shape

deformed target shape

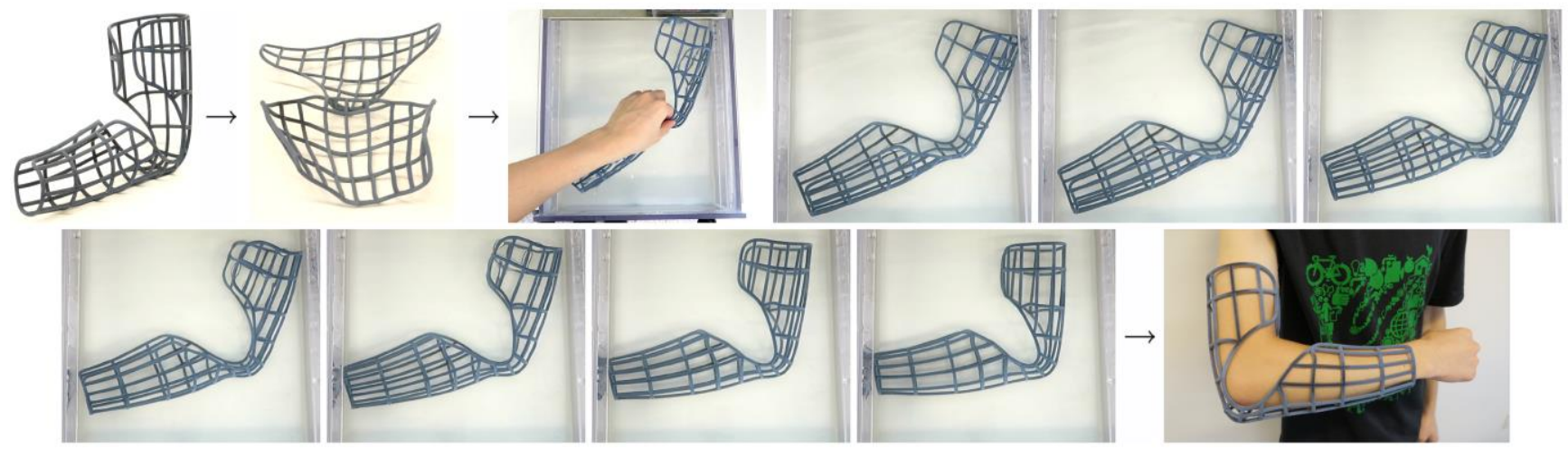

Figure 8: Arm cast. (a) A medical cast for the lower arm is optimized such that I can be manually flattened into its target configuration. From the target configuration, the cast can then actively deform back into the printed shape and fix a patient's arm. (b) Active shape recovery of the $3 \mathrm{D}$ printed cast.

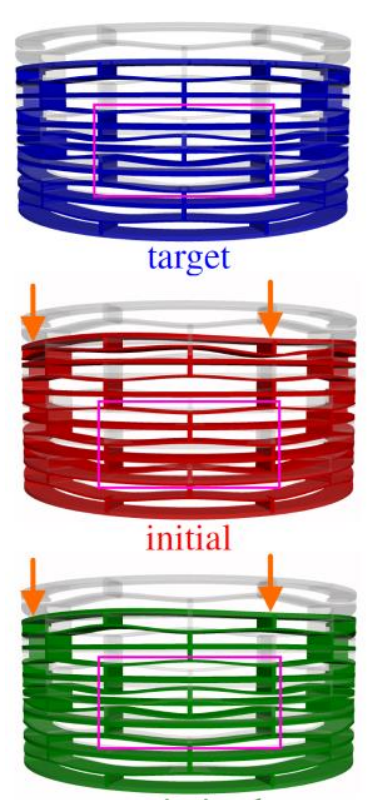

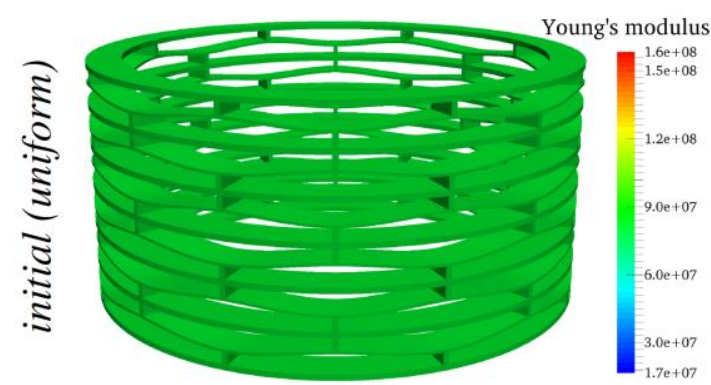

Young's modulus distribution

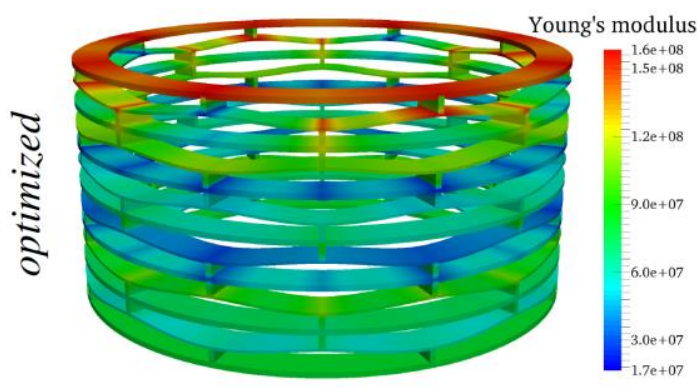

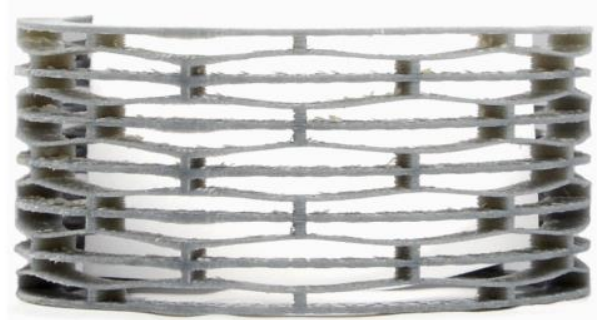

3D printed structure
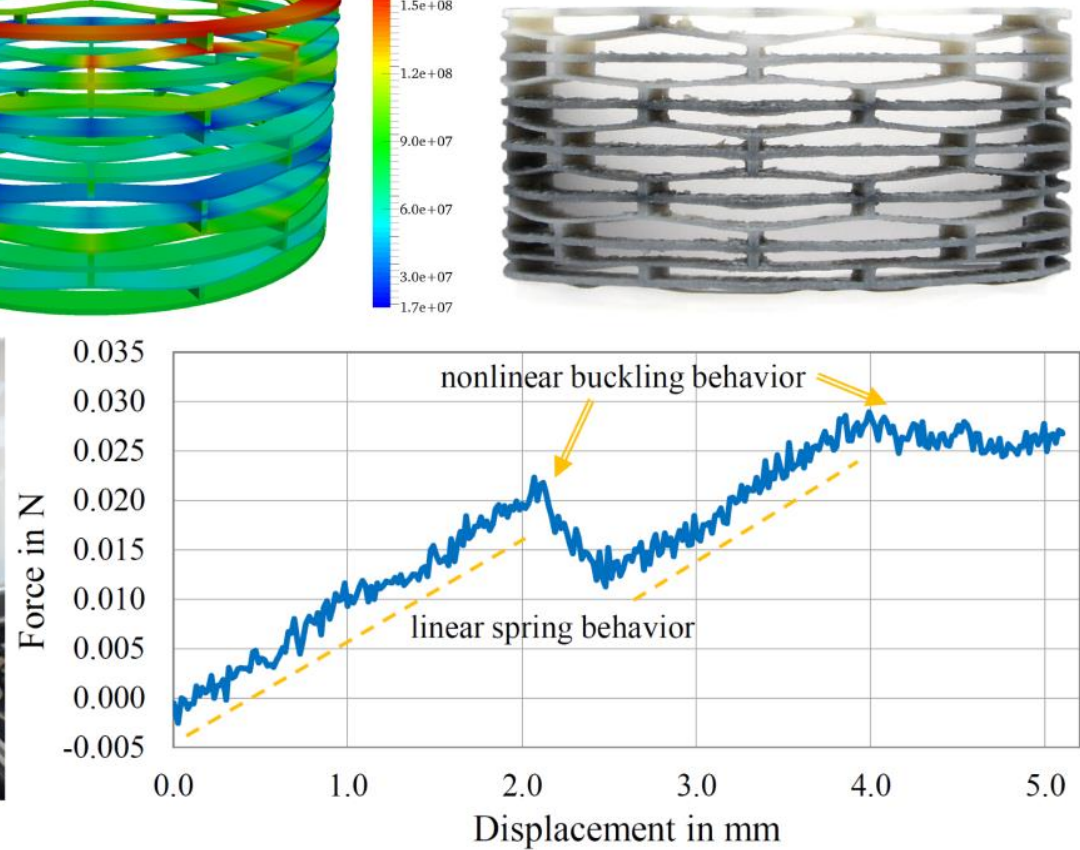

Figure 9: Buckling spring. (a) Comparison of uniform and optimized material distributions and their training behavior for computational and actual 3D printed models. (b) The compression test verifies that the buckling occurs in the vertical center of the optimized structure and the force-displacement curve of the optimized structure shows overall a highly nonlinear behavior due to the desired buckling effect. 


\section{Conclusion}

We have introduced a framework for the design and manufacture of 3D printed multi-material rod structures with active shape change behavior, so-called 4D printing. The integral components of our method are: the modeling and simulation of 3-dimensional rod structures; the formulation of a nonlinear optimization problem for assignment of spatially varying material distributions to match deformation of the rod structure during shape memory training phase with the target shape; and multi-material 3D printing stage to create realization of the rod structures with spatially varying, gradient elastic properties. Using several we have successfully validated the approach and demonstrated the desired training deformations and active shape recovery behavior.

A current limitation to the applicability of shape memory structures in our framework is the usage of hot and cold water baths to warm the rod structures for training deformation and cool them for shape storage. However, other methods such as using conductive heating by embedding electrical wires are already investigated. Furthermore, the active shape recovery behavior adopted in this work requires a training phase first. Considering other mechanical phenomena, such as thermal deformation and residual stresses, it could also possible to optimize the design of structures with a fully active behavior, which do not require training, can recover only gradually or even into different shapes.

\section{Acknowledgements}

The authors acknowledge support from the SUTD Digital Manufacturing and Design (DManD) Centre, supported by the Singapore National Research Foundation. We would like to thank Sawako Kaijima for helpful discussions, Zhen Ding for material measurements and Zhenghui Er for photo taking. This research was conducted on the Stratasys Connex 3 through Stratasys' Voxel Print Research Program, which enhances the value of 3D printing as a powerful platform for experimentation, discovery and innovation. For more information contact Stratasys Education, Academic Research and Development Unit: academic.research@stratasys.com.

\section{Author Disclosure Statement}

No competing financial interests exist.

\section{References}

1. Tibbits, S., Design to Self-Assembly. Arch Des 82 (2) (2012).

2. Ge, Q., Qi, H. J. \& Dunn, M. L., Active materials by four-dimension printing. Appl Phys Lett 103 (13) (2013).

3. Ge, Q., Dunn, C. K., Qi, H. J. \& Dunn, M. L., Active origami by 4D printing. Smart Mater Struct 23 (9) (2014).

4. Mao, Y. et al., Sequential Self-Folding Structures by 3D Printed Digital Shape Memory Polymers. Sci Rep 5 (2015).

5. Ge, Q. et al., Multimaterial 4D Printing with Tailorable Shape Memory Polymers. Sci Rep 6 (2016).

6.Zarek, M. et al., 3D Printing of Shape Memory Polymers for Flexible Electronic Devices. Adv Mater 18 (22) (2016).

7. Raviv, D. et al., Active Printed Materials for Complex Self-Evolving Deformations. Sci Rep (4) (2014).

8. Gladman, A. S., Matsumoto, E. A., Nuzzo, R. G., Mahadevan, L. \& Lewis, J. A., Biomimetic 4D printing. Nat Mater 15, 413-418 (2016).

9. Kwok, T.-H., Wang, C. C. L., Deng, D., Zhang, Y. \& Chen, Y., 4D printing for freeform surfaces: design optimization of Origami and Kirigami structures. J Mech Des 137 (11) (2015).

10. Maute, K. et al., Level Set Topology Optimization of Printed Active Composites. J Mech Des 137 (11) (2015).

11. Bader, C., Kolb, D., Weaver, J. C. \& Oxman, N., Data-Driven Material Modeling with Functional Advection for 3D Printing of Materially Heterogeneous Objects. 3D Print Addit Manuf 3 (2) (2016).

12. Chiu, W. K. \& Yu, K. M., Direct digital manufacturing of three-dimensional functionally graded material objects. Comput Aided Des 40 (2008).

13. Oxman, N., Dikovsky, D., Belocon, B. \& Carter, W. C., Gemini: Engaging Experiential and Feature Scales Through Multimaterial Digital Design and Hybrid Additive-Subtractive Fabrication. 3D Print Addit Manuf 1 (3) (2014).

14. Chen, X. et al., Dapper: decompose-and-pack for 3D printing. ACM Trans Graph 34 (6) (2015).

15. Vidimce, K., Wang, S.-P., Ragan-Kelley, J. \& Matusik, W., OpenFab: A Programmable Pipeline for Multimaterial Fabrication. ACM Trans Graph 32 (4) (2013). 
16. Hiller, J. \& Lipson, H., Dynamic Simulation of Soft Multimaterial 3D-Printed Objects. Soft Robotics 1 (1) (2014).

17. Hiller, J. \& Lipson, H., Design automation for multi-material printing. 20th Annual International Solid Freeform Fabrication Symposium (2009).

18. Hiller, J. D. \& Lipson, H., Multi Material Topological Optimization of Structures and Mechanisms. Proceedings of the 11th annual conference on genetic and evolutionary computation (2009).

19. Stankovic, T., Mueller, J., Egan, P. \& Shea, K., A Generalized Optimality Criteria Method for Optimization of Additively Manufactured Multi-Material Lattice Structures. J Mech Des 137 (11) (2015).

20. Perez, J. et al., Design and Fabrication of Flexible Rod Meshes. ACM Trans. Graph. 34 (4) (2015).

21. Antman, S. S., Nonlinear Problems of Elasticity (Springer, New York, 2005).

22. Weeger, O., Yeung, S.-K. \& Dunn, M. L., Isogeometric collocation methods for Cosserat rods and rod structures. Comput Methods in Appl Mech Eng (2016).

23. Hughes, T. J. R., Cottrell, J. A. \& Bazilevs, Y., Isogeometric analysis: CAD, finite elements, NURBS, exact geometry and mesh refinement. Comput Methods in Appl Mech Eng 194 (39-41) (2005).

24. Auricchio, F., Beirão da Veiga, L., Hughes, T. J. R., Reali, A. \& Sangalli, G., Isogeometric Collocation Methods. Math Models Methods Appl Sci 20 (11) (2010).

25. Garland, M. \& Heckbert, P. S., Surface Simplification Using Quadric Error Metrics, presented at Proceedings of the 24th Annual Conference on Computer Graphics and Interactive Techniques, Los Angeles, CA, USA, 1997 (unpublished).

26. Schwarz, M. \& Seidel, H.-P., Fast Parallel Surface and Solid Voxelization on GPUs. ACM Trans Graph 29 (6) (2010).

27. Cho, W., Sachs, E. M., Patrikalakis, N. M. \& Troxel, D. E., A dithering algorithm for local composition control with three-dimensional printing. Comput Aided Des 35 (9) (2003).

28. Doubrovski, E. L. et al., Voxel-based fabrication through material property mapping: A design method for bitmap printing. Comput Aided Des 60 (2015).

29. Ulichney, R., Digital Halftoning (MIT press, 1987).

Address correspondence to:

Oliver Weeger Singapore University of Technology and Design SUTD Digital Manufacturing and Design Centre

8 Somapah Road

Singapore 487372

Singapore

Email: oliver_weeger@sutd.edu.sg 